\title{
Survival rates of patients with tumors originating in different segments of the left upper lung in stage I to III non-small cell lung cancer
}

\author{
Yi Xiao" ${ }^{1 \#}$, Shaoning Luo ${ }^{2 \#}$, Jinyuan $\mathrm{He}^{1 \#}$, Yubin Zhou ${ }^{1}$, Wei Li ${ }^{1}$, Jun Lan ${ }^{3}$, Xiongwen Yang ${ }^{4}$, Shaohong Huang ${ }^{1}$ \\ ${ }^{1}$ Department of Thoracocardiac Surgery, The Third Affiliated Hospital of Sun Yat-sen University, Guangzhou, China; ${ }^{2}$ Department of Emergency \\ Medicine, The Third Affiliated Hospital of Sun Yat-sen University, Guangzhou, China; ${ }^{3}$ Department of General Surgical, The People's Hospital of \\ Gaoan City, Gaoan, China; ${ }^{4}$ Department of Oncological Surgery, The First People's Hospital of Chenzhou City, Chenzhou, China \\ Contributions: (I) Conception and design: Y Xiao, X Yang; (II) Administrative support: S Huang; (III) Provision of study materials or patients: X \\ Yang, Y Xiao, S Luo, J He; (IV) Collection and assembly of data: Y Zhou, W Li; (V) Data analysis and interpretation: Y Xiao, X Yang, J Lan; (VI) \\ Manuscript writing: All authors; (VII) Final approval of manuscript: All authors. \\ "These authors contributed equally to this work. \\ Correspondence to: Shaohong Huang. Department of Thoracocardiac Surgery, The Third Affiliated Hospital of Sun Yat-sen University, Tianhe \\ Road 600\#, Guangzhou 510630, China. Email: hshaoh@mail.sysu.edu.cn; Xiongwen Yang. Department of Oncological Surgery, The First People’s \\ Hospital of Chenzhou City, No. 102 Luojiajing Road, Chenzhou 423000, China. Email: yangxiongwen300@yeah.net.
}

\begin{abstract}
Background: The aim of this research was to evaluate the effect of spatial location of tumors on the prognosis of patients with left upper lung non-small cell lung cancer (NSCLC), with a focus on the S1+2+3 and lingual segment.

Methods: A total of 486 patients who underwent lobectomy and systematic lymph node dissection were collected retrospectively in this study (354 S1+2+3 and 132 lingual segment patients). Factors impacting survival were assessed via univariate analyses, multivariate analyses, and log-rank tests.

Results: Compared with tumor location in S1+2+3, lingual segment tumor location of stage II to III left upper lung NSCLC patients was significantly associated with a better 5-year disease-free survival (DFS) $(\mathrm{P}=0.041)$. Multivariate analysis results showed that tumor location in the lingual segment was a good independent prognostic factor of stage II to III left upper lung NSCLC patients [hazard ratio $(\mathrm{HR})=0.602$, 95\% confidence interval (CI): 0.149-0.865, $\mathrm{P}=0.006)$. However, in stage I left upper lung NSCLC, tumor location ( $\mathrm{HR}=1.069,95 \% \mathrm{CI}$ : 0.571-2.000, $\mathrm{P}=0.835$ ) was not an independent prognostic factor, and only $\mathrm{T} 2$ ( $\mathrm{HR}=2.422$, 95\% CI: 1.271-4.620, $\mathrm{P}=0.007)$ was an independent worse prognosis factor.

Conclusions: Tumor location in the lingual segment of left upper lung stage II to stage III NSCLC is a good independent prognostic factor compared with $\mathrm{S} 1+2+3$. Nevertheless, tumor location does not impact the prognosis of patients with stage I NSCLC in the left upper lung.
\end{abstract}

Keywords: Non-small cell lung cancer (NSCLC); left up lobe; survival rate; lingual segment; tumor location

Submitted Sep 09, 2021. Accepted for publication Oct 20, 2021.

doi: $10.21037 /$ atm-21-5157

View this article at: https://dx.doi.org/10.21037/atm-21-5157

\section{Introduction}

Lung cancer is one of the main causes of cancer-related death worldwide $(1,2)$. Recently, the American Cancer Society predicted that in 2021 , there will be 235,760 new lung cancer patients and 131,880 lung cancer deaths in the
United States (3). Undoubtedly, surgery is the best and most crucial method for curing most lung cancers, except for patients who cannot benefit from surgery. In terms of anatomy, lobes are divided into different segments by different intersegmental veins. The left upper lung (LUL) 
consists of 3 segments: apicoposterior segment $(\mathrm{S} 1+2)$, anterior segment (S3), and lingual segment $(\mathrm{S} 4+5)$. The combination of the apicoposterior segment and anterior segment is called $\mathrm{S} 1+2+3$.

Previous research has shown that the location of tumor origin is related to the prognosis of patients. Handa and colleagues reported that the spatial location of the tumor, including different segments in the lower lobe, was a prognostic factor of patients with stage I non-small cell lung cancer (NSCLC), and tumors originating in the dorsal segment had a poorer prognosis than tumors in the basal segment (4). The researchers speculated that the different prognoses caused by the different lung segment tumors might be related to the location of the lung segment from the mediastinal lymph nodes (5). The impact of tumor origin from different lobes on prognosis has also been investigated. The location of the tumor was also a prognostic factor in patients had non-surgical treatment, previous study revealed that primary tumor in the lower lung was related to the poor prognosis of patients received stereotactic body radiation therapy (SBRT) (6). Meanwhile, previous studies have shown that the prognosis of right middle lung tumors was worse when compared with other right-side tumors (7). Nonetheless, most articles studied the impact of tumors location on the prognosis of patients were focus on tumors in different lobes. There is lack of research on the prognosis of tumors that originate from different segments of the longest lobe, the LUL. Therefore, this study was focused on exploring the impact of tumors originating from different segments of the LUL on the prognosis of patients.

The primary purpose of this study was to investigate the discrepancy between the prognosis of patients with LUL tumors originating from the $\mathrm{S} 1+2+3$ and lingual segment. The second aim was to analyze the significant factors that affect the prognosis of LUL cancer patients. The primary research endpoint was the 5 -year disease-free survival (DFS) rate. We present the following article in accordance with the STROBE reporting checklist (available at https://dx.doi. org/10.21037/atm-21-5157).

\section{Methods}

\section{Patient population}

All procedures performed in this study involving human participants were in accordance with the Declaration of Helsinki (as revised in 2013). This retrospective study was approved by the ethics board of The Third Affiliated Hospital of Sun Yat-sen University (No. [2020]02540) and The First People's Hospital of Chen Zhou city (No. CY2020NO.0037). Individual consent for this retrospective analysis was waived. Between January 2010 and January 2016, the clinical data of 486 patients who were diagnosed with NSCLC by postoperative pathology and underwent lobectomy and systematic lymph node dissection were collected, including gender, age, smoking history, tumor location, tumor grade, tumor histology, surgical method, pTNM (8th edition) (8), postoperative treatment, and DFS. The inclusion and exclusion criteria are shown in Figure 1. The location of the tumor was determined by imaging data. More than $70 \%$ of the tumor volume located in the $\mathrm{S} 1+2+3$ or tongue segment was defined as location determination if tumor was cross the intersegmental. The results of bronchoscopy indicated that the tumor invading the main lobe bronchus was considered to be of uncertain lung segment origin.

\section{Follow-up}

Patients who were diagnosed with NSCLC by pathology were recommended to undergo reexamination every 6 months within 3 years after surgery, and once a year thereafter. In general, the evaluation of patients with stage I NSCLC included chest computed tomography (CT) scanning and physical examination, and stage II to stage III patients were required to undergo additional brain magnetic resonance imaging and tumor marker examinations. Radionuclide bone scanning was not recommended unless the patient requested it or appeared to have specific symptoms. DFS was defined as the time from surgery treatment to tumor recurrence or death.

\section{Statistical analysis}

All statistical analysis were performed using R software v3.61 (https://www.r-project.org/). Classification variables included smoking history, tumor location, tumor grade, tumor stage, and survival status. Continuous variables included age and DFS. According to the cut-off point (65 years old), age was transformed into a categorical variable. In this study, followup information was missing in 32 patients. Subsequently, these patients were removed. To investigate the effect of the spatial location of the tumor on the prognosis of different stage patients with LUL NSCLC, patients were classified into 2 groups (stage I and stage II/III). The Pearson $\chi^{2}$ test 


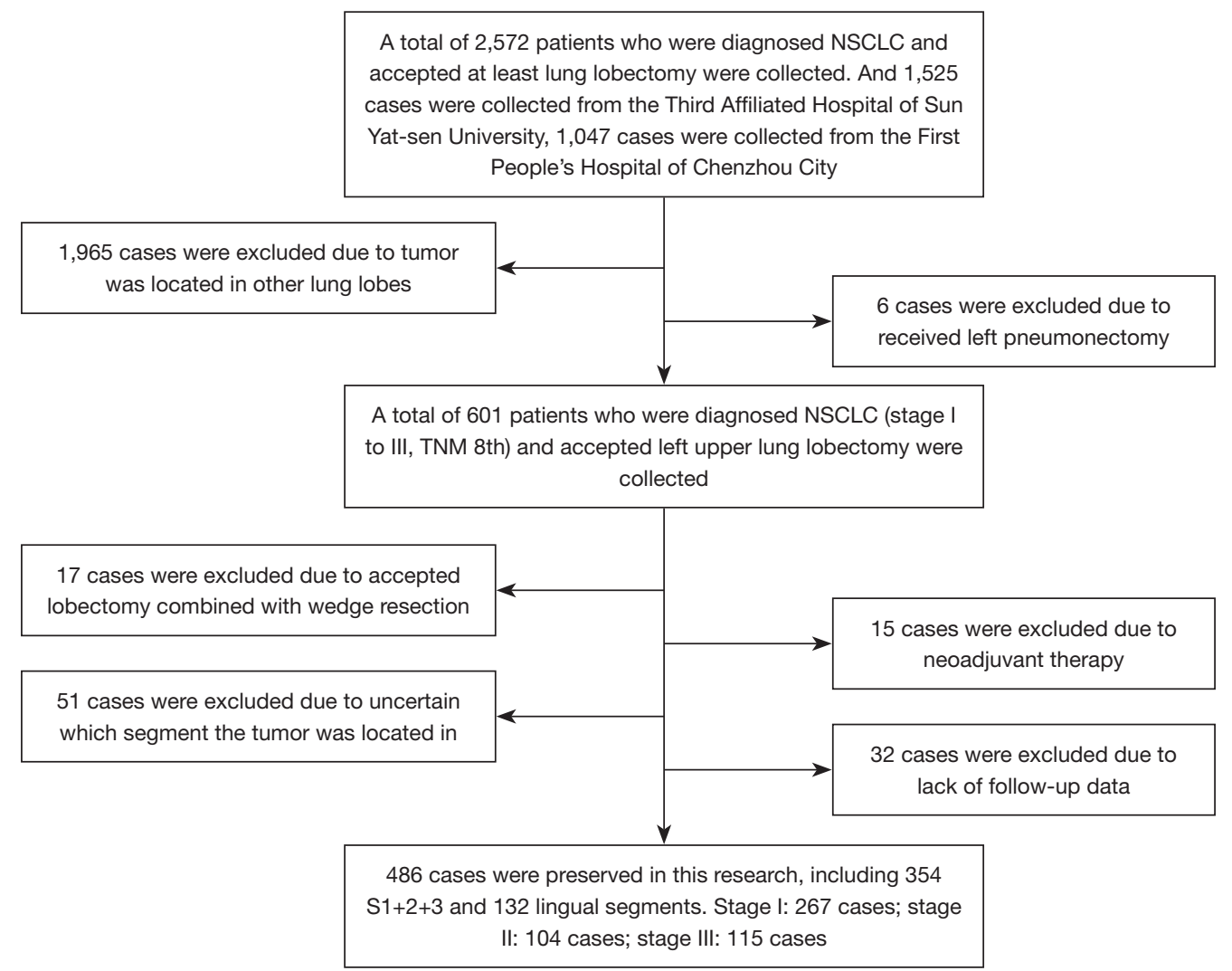

Figure 1 Flow-chart of inclusion and exclusion criteria. The tumor that located in the intersegmental plane and more than $70 \%$ located in $\mathrm{S} 1+2+3$ or tongue segment was defined as location determination. And results of bronchoscopy indicated that squamous cell carcinoma invading the main lobe bronchus was considered to be of uncertain lung segment. NSCLC, non-small cell lung cancer.

and Fisher's exact test were used to determine significant differences between groups for categorical variables. DFS curves were generated using the Kaplan-Meier method and assessed by the log-rank test. The influence of tumor location in the tongue segment or $\mathrm{S} 1+2+3$ on prognosis was assessed by univariate cox regression analysis and multivariate cox regression analysis. All $\mathrm{P}$ values in this study were two-sided, and $\mathrm{P}<0.05$ was considered statistically significant.

\section{Results}

\section{Patient characteristics and prognosis concerning the $S 1+2+3$ and lingual segment (stage I to III)}

A total of 486 patients were included in the study. Table 1 summarizes the general clinical characteristics of patients. Of these patients, $50.4 \%(\mathrm{n}=245)$ were male and $49.6 \%$ were female $(n=241)$. Additionally, $27.2 \%$ of the tumors originated from the tongue $(\mathrm{n}=132)$ and $72.8 \%$ originated from $\mathrm{S} 1+2+3(\mathrm{n}=354)$. There were $54.9 \%$ of patients with stage I NSCLC $(n=267), 21.4 \%$ patients with stage II NSCLC $(n=104)$, and $23.7 \%$ patients with stage III NSCLC ( $\mathrm{n}=115)$. The median follow-up for all patients in this research was 42.2 months. The percentages of the 5 -year DFS of patients with LUL S1+2+3 and lingual segment were $43.5 \%$ and $41.7 \%$, respectively. Tumor grade was missing in 50 patients $(50 / 486,10.3 \%)$. The results of the log-rank test $(\mathrm{P}=0.36)$ showed that the prognosis of patients with $\mathrm{S} 1+2+3$ tumors was not significantly different compared with that of patients with stage I to III lingual segment tumors (Figure 2).

\section{Univariate and multivariate analyses (stage I to III)}

The prognostic factors of patients with LUL NSCLC were investigated using Cox regression analysis. The results of the univariate Cox regression analysis showed that age, 
Table 1 General clinicopathological characteristics of patient

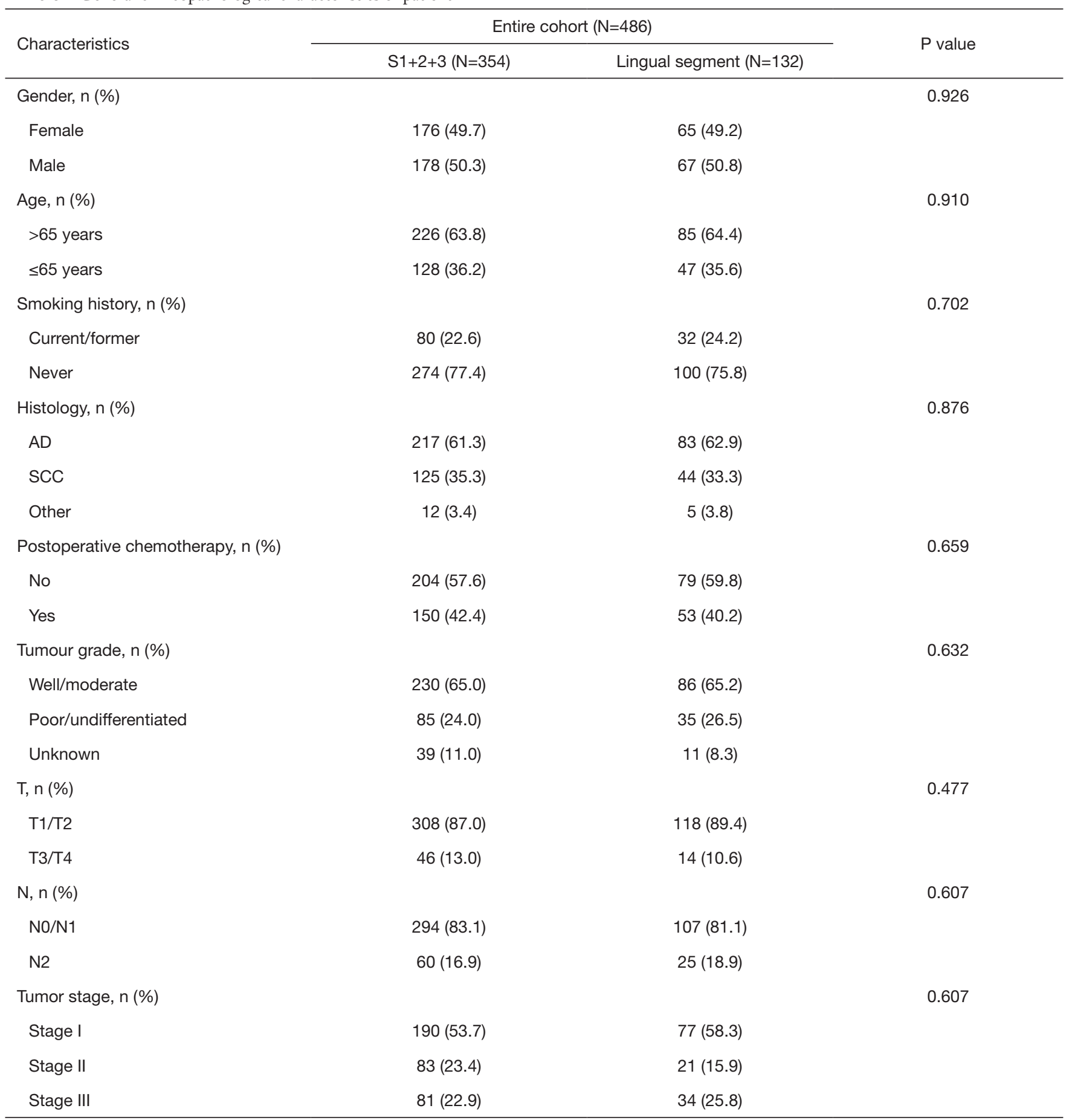

$A D$, adenocarcinoma; SSC, squamous cell carcinoma.

tumor location, lymph node metastasis, postoperative chemotherapy, tumor grade, and $\mathrm{T}$ stage were the independent prognostic factors of patients with stage I, II, and III LUL NSCLC. Age higher than 65 was associated with worse DFS [hazard ratio $(\mathrm{HR})=0.679,95 \%$ confidence interval (CI): $0.505-0.914, \mathrm{P}=0.011]$. Tumors in the lingual segment indicated significantly better DFS $(\mathrm{HR}=0.679$, 95\% CI: $0.530-0.969, \mathrm{P}=0.030$ ). The subsequent results of 


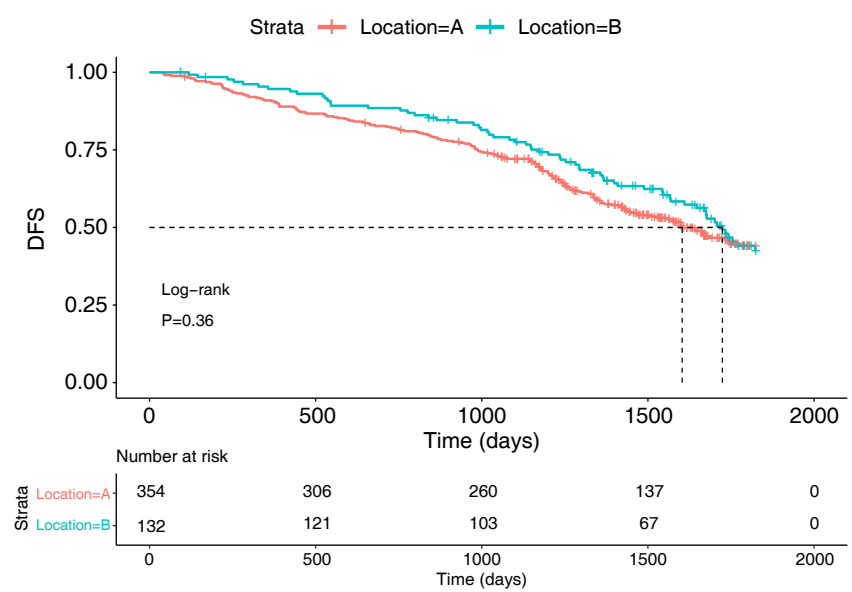

Figure 2 DFS curves for stage I to stage III patients with tumors located in $\mathrm{S} 1+2+3$ and lingual segment. Location A and B refer to $S 1+2+3$ and lingual segment, respectively. DFS, disease-free survival.

the multivariate Cox regression analysis showed that age (HR $=0.679,95 \%$ CI: $0.505-0.914 \mathrm{P}=0.010$ ), tumor location (HR $=0.717,95 \%$ CI: $0.530-0.969 \mathrm{P}=0.030), \mathrm{N} 2$ metastasis (HR $=2.008$, 95\% CI: 1.099-3.670, P=0.024), and tumor stage were the independent prognostic factors (Table 2).

\section{Patient characteristics and prognosis concerning the $S 1+2+3$ and lingual segment (stage I)}

The number of stage I patients was 267 (IA =202, IB =65), accounting for $54.9 \%$ of the total patients. Figure 3 surveys the general clinical characteristics of the stage I patients. Stage I patients included 127 (47.6\%) males and 140 (52.4\%) females. A total of $71.2 \%$ of the tumors originated from the $\mathrm{S} 1+2+3(\mathrm{n}=190)$, and $28.8 \%$ originated from the lingual segment $(n=77)$. The percentages of the 5 -year DFS of patients with LUL S1+2+3 and lingual segment were $76.4 \%$ and $65.7 \%$, respectively. The log-rank test demonstrated that the 5 -year DFS of patients with $\mathrm{S} 1+2+3$ and lingual segment tumors was not significantly different (Figure 4, $\mathrm{P}=0.16$ ).

\section{Multivariate analyses (stage I)}

The results of the univariate cox regression analysis indicated that age $(\mathrm{P}=0.520)$, tumor location $(\mathrm{P}=0.835)$, postoperative chemotherapy $(\mathrm{P}=0.434)$, and tumor grade $(\mathrm{P}=0.383)$ were not independent prognostic factors of patients with stage I LUL NSCLC. Only T2 (HR =2.422,
95\% CI: $1.271-4.620, \mathrm{P}=0.007)$ was an independent worse prognosis factor.

\section{Patient characteristics and prognosis concerning the $S 1+2+3$ and lingual segment (stage II and III)}

A total of 219 patients were diagnosed with stage II and III NSCLC in the study. Figure 2 summarizes the general clinical characteristics of patients. The number of patients with tumors located in the $\mathrm{S} 1+2+3$ and lingual segment was 164 (74.9\%) and 55 (25.1\%), respectively. Additionally, $46.1 \%$ of the patients $(\mathrm{n}=101)$ were female and $53.9 \%$ were male $(n=118)$. There were $47.5 \%$ of patients with stage II $(\mathrm{n}=104)$ and $52.5 \%$ of patients with stage III NSCLC. Kaplan-Meier survival analysis and the log-rank test indicated that the percentages of the 5-year DFS of patients with LUL S1+2+3 and lingual segment NSCLC were 9.3\% and $13.1 \%$, respectively. The log-rank test comparison revealed that compared with tumors in the $\mathrm{S} 1+2+3$, lingual segment tumors were significantly associated with a better 5-year DFS (Figure 5, P=0.041).

\section{Multivariate analyses (stage II and stage III)}

Multivariate analysis results indicated that the 5-year DFS was worse for patients with $\mathrm{S} 1+2+3$ tumors compared with lingual segment tumors (HR $=0.602,95 \%$ CI: 0.149-0.865, $\mathrm{P}=0.006$ ) (Figure 6). Other poor prognostic factors of patients with LUL stage II and stage III NSCLC were age higher than 65 years old (HR $=0.639,95 \%$ CI: $0.446-0.915$, $\mathrm{P}=0.0145)$ and $\mathrm{N} 2(\mathrm{P}<0.001)$.

\section{Discussion}

Over the past three decades, lung adenocarcinoma had gradually replaced squamous cell carcinoma to become the most common histological type, especially in nonsmoking patients $(9,10)$. In this study, $300(61.7 \%)$ patients diagnosed with lung adenocarcinoma and 169 (34.8\%) patients diagnosed with lung squamous cell carcinoma were included. The probability of tumors originating from the tongue segment was lower than that for $\mathrm{S} 1+2+3$, with a ratio of about $1: 3$. Although the reason is not clear, considering that the volume of the $\mathrm{S} 1+2+3$ is larger than that of the tongue segment, the volume may be one of the main reasons for the different probabilities of tumor occurrence in different segments. Previous research has shown that the right middle lung has the minimum incidence of tumorigenesis of all lobes, accounting for $3.8 \%$ to $6.7 \%$ of 
Table 2 Characteristics of patients and results of univariate and multivariate analyses

\begin{tabular}{|c|c|c|c|c|c|c|c|}
\hline Characteristic & Number (\%) & \multicolumn{3}{|c|}{ Univariate } & \multicolumn{3}{|c|}{ Multivariate } \\
\hline \multicolumn{8}{|l|}{ Gender } \\
\hline Female & 241 (49.5) & Ref. & & & Ref. & & \\
\hline Male & $245(50.5)$ & 1.114 & $0.863-1.438$ & 0.405 & 1.162 & $0.884-1.526$ & 0.284 \\
\hline$>65$ years & 311 (63.9) & Ref. & & & Ref. & & \\
\hline$\leq 65$ years & $175(36.1)$ & 0.5147 & $0.387-0.685$ & $<0.001$ & 0.679 & $0.505-0.914$ & 0.011 \\
\hline \multicolumn{8}{|l|}{ Tumor location } \\
\hline $\mathrm{S} 1+2+3$ & 354 (72.9) & Ref. & & & Ref. & & \\
\hline Well/moderate & $316(65.2)$ & Ref. & & & Ref. & & \\
\hline Poor/undifferentiated & $120(24.6)$ & 1.633 & $1.2294-2.168$ & $<0.001$ & 1.103 & $0.790-1.539$ & 0.567 \\
\hline Unknown & $50(10.2)$ & 1.149 & $0.7508-1.760$ & 0.521 & 1.351 & $0.865-2.112$ & 0.186 \\
\hline \multicolumn{8}{|l|}{ Smoking history } \\
\hline No & $374(76.1)$ & Ref. & & & Ref. & & \\
\hline Yes & $112(23.9)$ & 0.815 & $0.592-1.257$ & 0.209 & 0.744 & $0.606-1.212$ & 0.383 \\
\hline \multicolumn{8}{|l|}{ Histology } \\
\hline$A D$ & $300(61.7)$ & Ref. & & & Ref. & & \\
\hline \multicolumn{8}{|l|}{$\mathrm{T}$} \\
\hline $\mathrm{T} 1 / \mathrm{T} 2$ & $417(85.8)$ & Ref. & & & Ref. & & \\
\hline $\mathrm{T} 3 / \mathrm{T} 4$ & $69(14.2)$ & 2.848 & $2.088-3.885$ & $<0.001$ & 1.429 & $0.921-2.218$ & 0.111 \\
\hline \multicolumn{8}{|l|}{$N$} \\
\hline No/N1 & 401 (82.6) & Ref. & & & Ref. & & \\
\hline N2 & 85 (17.4) & 5.897 & $4.480-7.760$ & $<0.001$ & 2.008 & $1.099-3.670$ & 0.024 \\
\hline \multicolumn{8}{|l|}{ Tumor stage } \\
\hline Stage I & 267 (54.9) & Ref. & & & Ref. & & \\
\hline Stage II & $104(21.4)$ & 7.233 & $5.053-10.350$ & $<0.001$ & 5.187 & $2.847-9.451$ & $<0.001$ \\
\hline Stage III & $115(23.7)$ & 14.039 & $10.012-19.690$ & $<0.001$ & 6.024 & $2.574-14.100$ & $<0.001$ \\
\hline
\end{tabular}

$A D$, adenocarcinoma; SSC, squamous cell carcinoma 


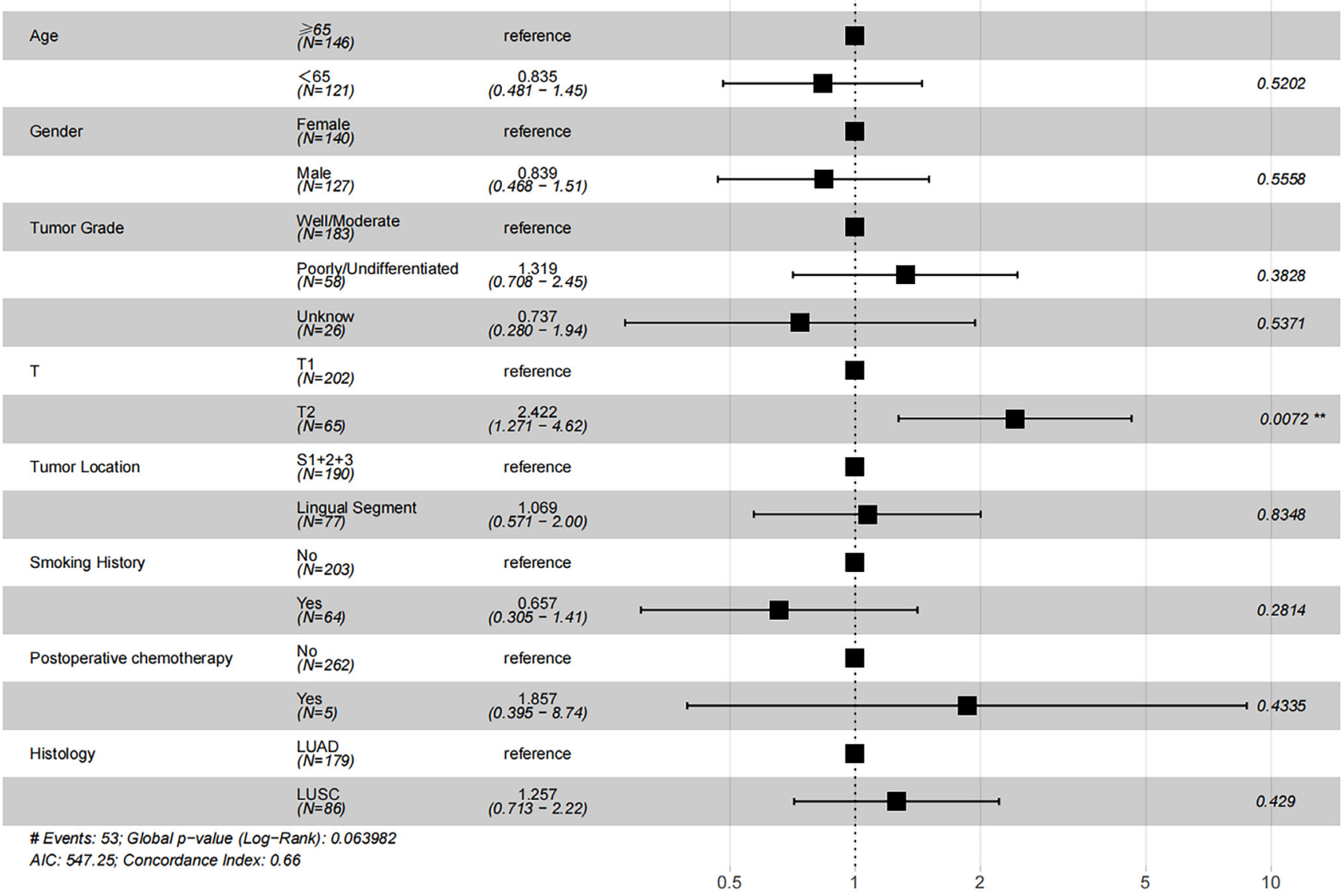

Figure 3 Multivariable Cox regression analysis of patients with stage I left upper lung non-small cell lung cancer. **, P<0.01. LUAD, lung adenocarcinoma; LUSC, lung squamous cell carcinoma; AIC, Akaike's information criterion.

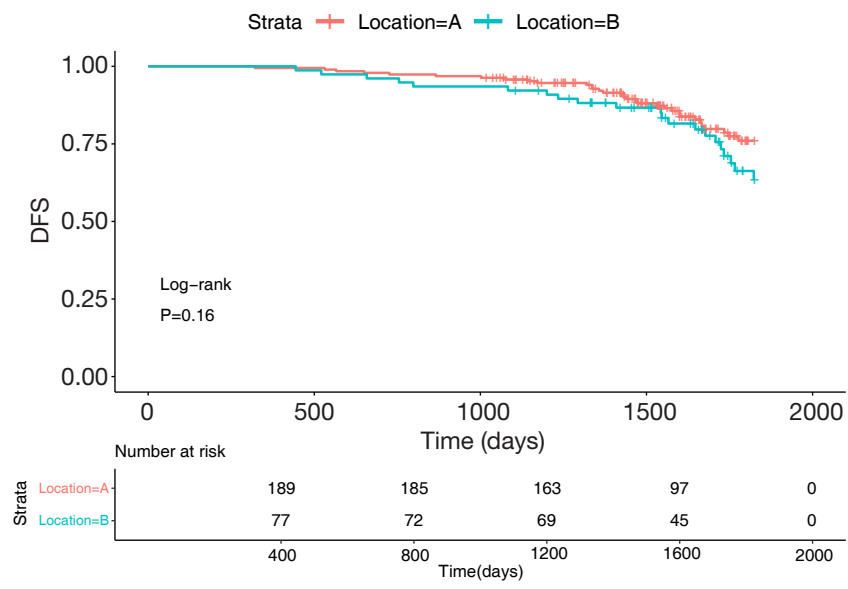

Figure 4 DFS curves for stage I patients with tumors located in $\mathrm{S} 1+2+3$ and lingual segment. Location $\mathrm{A}$ and $\mathrm{B}$ refer to $\mathrm{S} 1+2+3$ and lingual segment, respectively. DFS, disease-free survival.

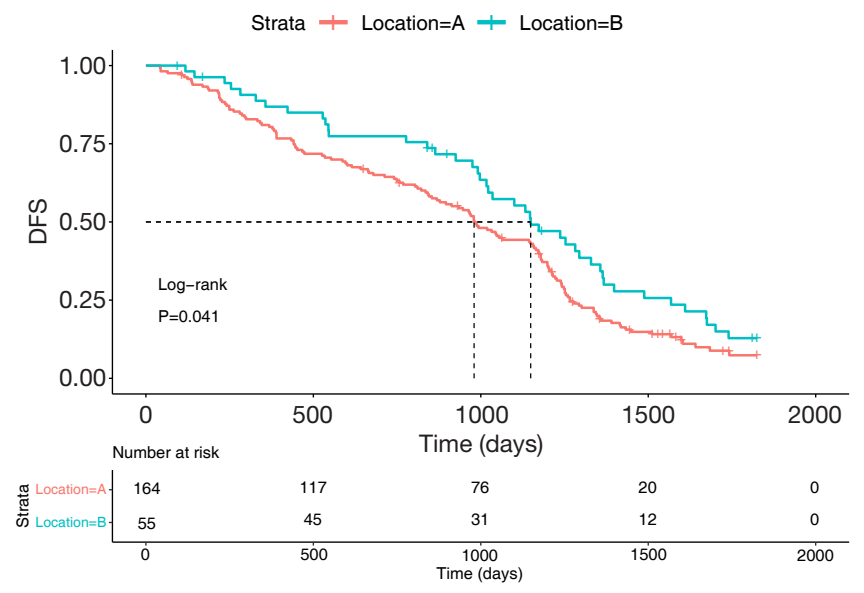

Figure 5 DFS curves for stage II and III patients with tumors located in $\mathrm{S} 1+2+3$ and lingual segment. Location A and B refer to $\mathrm{S} 1+2+3$ and lingual segment, respectively. DFS, disease-free survival. 


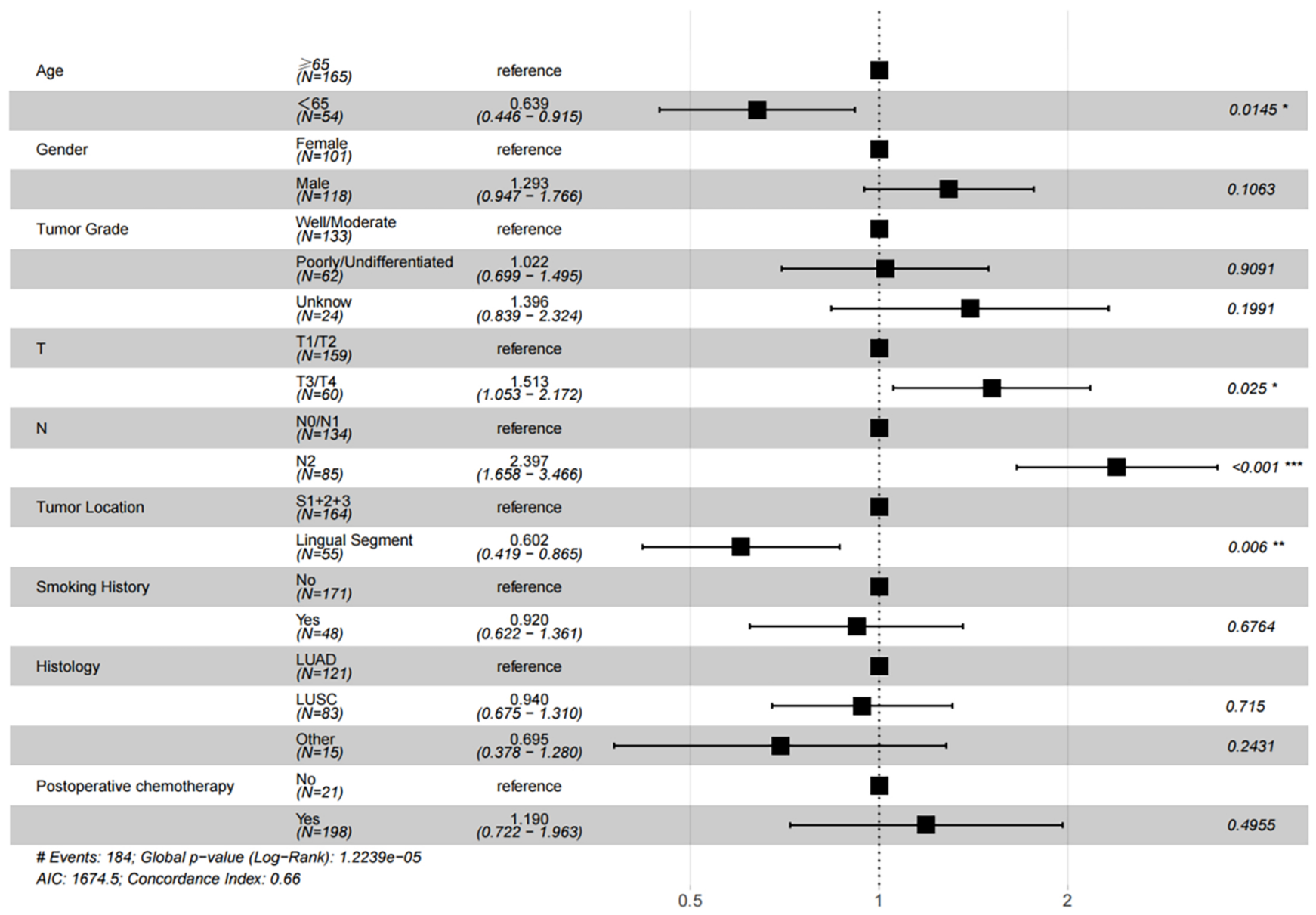

Figure 6 Multivariable Cox regression analysis of patients with stage II and III of left upper lung non-small cell lung cancer. *, P<0.05; **, $\mathrm{P}<0.01$; **, $\mathrm{P}<0.001$. LUAD, lung adenocarcinoma; LUSC, lung squamous cell carcinoma; AIC, Akaike's information criterion.

all lung cancers, which also validates this speculation (11).

The results of the log-rank test $(\mathrm{P}=0.36)$ in this study showed that the prognosis of patients with $\mathrm{S} 1+2+3$ tumors was not significantly different compared with that of patients with stage I to III lingual segment tumors. However, multivariate analyses showed that the tumor location is an independent prognosis factor of stage I to stage III patients. Subsequently, patients were divided into 2 groups according to their pathological stage: the stage I group and the stage II/III group. In the stage I group, the log-rank test results showed that the 5 -year DFS between the $\mathrm{S} 1+2+3$ and lingual segment was not significantly different $(\mathrm{P}=0.16)$. Then, multivariate analysis was carried out, and the outcomes confirmed that tumor location was not an independent prognostic factor, only T2 $(\mathrm{HR}=2.422,95 \% \mathrm{CI}: 1.271-4.620, \mathrm{P}=0.007)$ was an independent worse prognosis factor in the stage I group. Besides, the study of molecular and pathological classification of lung cancer makes the understanding of the factors affecting the prognosis of early lung cancer more in-depth. Integration protein genomics studies suggest that protein expression differences in early lung adenocarcinoma are quite important prognostic factors (12). In addition, the difference of pathological subtypes is also important for the prognosis of lung adenocarcinoma (13). In the stage II and III group, tumors located in the lingual segment showed a better prognosis (HR $=0.602,95 \% \mathrm{CI}$ : $0.419-0.865, \mathrm{P}=0.006$ ), and the log-rank test demonstrated that the 5-year DFS of patients with tumors located in the lingual segment was better compared with tumor origin in the $S 1+2+3$. Previous studies have reported the impact of tumor location on the prognosis of patients. Puri and colleagues previously reported that both the 3-year and 5 -year survival rates between different lobes of stage I and 
II NSCLC patients were not significantly different (14). Shan $e t a l$. indicated that the location of the tumor may affect the prognosis of patients with stage IV NSCLC, and primary tumors originating in different lobes had various trends in metastasizing to different organs. Upper lobe tumors more easily metastasized to the brain, middle lobe tumors were more likely to lead to lung metastasis, and lower lobe tumors tended to result in bone metastasis and lung metastasis (15). Ketchedjian and colleagues reported that tumors in the superior segment had a higher potential to induce mediastinal metastasis compared with those from the basal segment ( $45 \%$ vs. $17 \%, \mathrm{P}=0.0003$ ), and there was a significant difference in prognosis between superior segment and basal segment tumors only when tumors were of $\mathrm{N} 2$ status (16). Handa et al. reported that the basal segment was a good prognostic factor compared with the superior segment ( $\mathrm{HR}=2.90,95 \% \mathrm{CI}: 1.20-7.00, \mathrm{P}=0.008$ ), and had better 5 -year recurrence-free survival $(54.4 \% \mathrm{vs}$. $75.7 \%, \mathrm{P}=0.032$ ) (4). Handa and colleagues demonstrated that tumors located in different segments of the right middle lung (S4 and S5) had a significant difference, and the main cause of the poor prognosis of S5 was presumed to be the shorter distance to the mediastinal lymph nodes (17).

Interestingly, as the longest lobe, the LUL extends almost from the top of the chest to the diaphragm, and the distance between tumors that are located in the lingual segment to the mediastinal lymph nodes is evidently longer than that of $\mathrm{S} 1+2+3$. Thus, we speculate that one of the important reasons explaining why tumors originating from the lingual segment had a better prognosis was the degree of difficulty of metastasis to the mediastinal lymph nodes, which may be determined by the distance to the hilar and mediastinal lymph nodes in stage II and III LUL NSCLC. The result that tumor location was not an independent risk factor in stage I seems to support this speculation. Beyond that, Rocha et al. indicated that tumors which originate from the lower lobe were worse due to upstaging after surgery (18). Hayakawa and colleagues reported that the various lobe tumors had different prognoses because of the difficulty grade of surgical treatment, as more obstacles were faced during the treatment of lower lobe tumors compared with upper lobe tumors (19). EGFR mutations in different lung lobes have also been studied. Some research demonstrated that the rate of EGFR-positive tumors was higher in upper lung tumors, which may affect the followup treatment and prognosis of patients $(20,21)$. It can be concluded that the tumor location, including different lobes and different segments, is an independent prognostic factor, and the reasons may be various.

The limitation of this study was that in order to avoid the impact of different surgical methods on the results, many patients with squamous cell carcinoma who underwent sleeve resection were excluded.

\section{Conclusions}

Tumor location in the lingual segment of LUL stage II to stage III NSCLC patients is a good independent prognostic factor compared with $\mathrm{S} 1+2+3$. Nevertheless, tumor location does not affect the prognosis of patients with stage I LUL NSCLC.

\section{Acknowledgments}

Funding: This research was supported by Guangdong Natural Science Foundation (No. 2021A1515012548).

\section{Footnote}

Reporting Checklist: The authors have completed the STROBE reporting checklist. Available at https://dx.doi. org/10.21037/atm-21-5157

Data Sharing Statement: Available at https://dx.doi. org/10.21037/atm-21-5157

Conflicts of Interest: All authors have completed the ICMJE uniform disclosure form (available at https://dx.doi. org/10.21037/atm-21-5157). The authors have no conflicts of interest to declare.

Ethical Statement: The authors are accountable for all aspects of the work in ensuring that questions related to the accuracy or integrity of any part of the work are appropriately investigated and resolved. All procedures performed in this study involving human participants were in accordance with the Declaration of Helsinki (as revised in 2013). This retrospective study was approved by the ethics board of The Third Affiliated Hospital of Sun Yatsen University (No. [2020]02-540) and The First People's Hospital of Chen Zhou city (No. CY2020NO.0037). Individual consent for this retrospective analysis was waived.

Open Access Statement: This is an Open Access article distributed in accordance with the Creative Commons Attribution-NonCommercial-NoDerivs 4.0 International 
License (CC BY-NC-ND 4.0), which permits the noncommercial replication and distribution of the article with the strict proviso that no changes or edits are made and the original work is properly cited (including links to both the formal publication through the relevant DOI and the license). See: https://creativecommons.org/licenses/by-nc-nd/4.0/.

\section{References}

1. Jemal A, Siegel R, Ward E, et al. Cancer statistics, 2009. CA Cancer J Clin 2009;59:225-49.

2. Chen $W$, Zheng R, Baade PD, et al. Cancer statistics in China, 2015. CA Cancer J Clin 2016;66:115-32.

3. Siegel RL, Miller KD, Fuchs HE, et al. Cancer Statistics, 2021. CA Cancer J Clin 2021;71:7-33.

4. Handa Y, Tsutani Y, Tsubokawa N, et al. Clinical Prognosis of Superior Versus Basal Segment Stage I Non-Small Cell Lung Cancer. Ann Thorac Surg 2017;104:1896-901.

5. Nishio W, Yoshimura M, Maniwa Y, et al. Re-Assessment of Intentional Extended Segmentectomy for Clinical T1aN0 Non-Small Cell Lung Cancer. Ann Thorac Surg 2016;102:1702-10.

6. Shaverdian N, Veruttipong D, Wang J, et al. Location Matters: Stage I Non-Small-cell Carcinomas of the Lower Lobes Treated With Stereotactic Body Radiation Therapy Are Associated With Poor Outcomes. Clin Lung Cancer 2017;18:e137-42.

7. Mazza F, Ferrari E, Maineri P, et al. Pulmonary middle lobectomy for non-small-cell lung cancer: effectiveness and prognostic implications. Eur J Cardiothorac Surg 2015;48:e117-23.

8. Goldstraw P, Chansky K, Crowley J, et al. The IASLC Lung Cancer Staging Project: Proposals for Revision of the TNM Stage Groupings in the Forthcoming (Eighth) Edition of the TNM Classification for Lung Cancer. J Thorac Oncol 2016;11:39-51.

9. Samet JM. Is the Incidence of Adenocarcinoma of the Lung Rising in Never Smokers? J Natl Cancer Inst 2017.

10. National Lung Screening Trial Research Team. Lung Cancer Incidence and Mortality with Extended Follow-

Cite this article as: Xiao Y, Luo S, He J, Zhou Y, Li W, Lan J, Yang X, Huang S. Survival rates of patients with tumors originating in different segments of the left upper lung in stage I to III non-small cell lung cancer. Ann Transl Med 2021;9(20):1590. doi: 10.21037/atm-21-5157 up in the National Lung Screening Trial. J Thorac Oncol 2019;14:1732-42.

11. Vincent RG, Takita H, Lane WW, et al. Surgical therapy of lung cancer. J Thorac Cardiovasc Surg 1976;71:581-91.

12. Xu JY, Zhang C, Wang X, et al. Integrative Proteomic Characterization of Human Lung Adenocarcinoma. Cell 2020;182:245-261.e17.

13. Moreira AL, Ocampo PSS, Xia Y, et al. A Grading System for Invasive Pulmonary Adenocarcinoma: A Proposal From the International Association for the Study of Lung Cancer Pathology Committee. J Thorac Oncol 2020;15:1599-610.

14. Puri V, Garg N, Engelhardt EE, et al. Tumor location is not an independent prognostic factor in early stage nonsmall cell lung cancer. Ann Thorac Surg 2010;89:1053-9.

15. Shan Q, Li Z, Lin J, et al. Tumor Primary Location May Affect Metastasis Pattern for Patients with Stage IV NSCLC: A Population-Based Study. J Oncol 2020;2020:4784701.

16. Ketchedjian A, Daly BD, Fernando HC, et al. Location as an important predictor of lymph node involvement for pulmonary adenocarcinoma. J Thorac Cardiovasc Surg 2006;132:544-8.

17. Handa Y, Tsutani Y, Ikeda T, et al. Reassessment of Right Middle Lobe Lung Cancer: Comparison of Segments 4 and 5 Tumors. Ann Thorac Surg 2018;105:1543-50.

18. Rocha AT, McCormack M, Montana G, et al. Association between lower lobe location and upstaging for early-stage non-small cell lung cancer. Chest 2004;125:1424-30.

19. Hayakawa K, Mitsuhashi N, Saito Y, et al. Impact of tumor extent and location on treatment outcome in patients with stage III non-small cell lung cancer treated with radiation therapy. Jpn J Clin Oncol 1996;26:221-8.

20. Kinsey CM, Estepar RS, Zhao Y, et al. Invasive adenocarcinoma of the lung is associated with the upper lung regions. Lung Cancer 2014;84:145-50.

21. Tseng CH, Chen KC, Hsu KH, et al. EGFR mutation and lobar location of lung adenocarcinoma. Carcinogenesis 2016;37:157-62.

(English Language Editor: C. Betlzar) 